\title{
Emoji Accessibility for Visually Impaired People
}

\author{
Garreth W. Tigwell \\ Rochester Institute of Technology \\ Rochester, NY, USA \\ garreth.w.tigwell@ rit.edu
}

\author{
Benjamin M. Gorman \\ Bournemouth University \\ Bournemouth, England, UK \\ bgorman@bournemouth.ac.uk
}

\author{
Rachel Menzies \\ University of Dundee \\ Dundee, Scotland, UK \\ r.menzies@dundee.ac.uk
}

\begin{abstract}
Emoji are graphical symbols that appear in many aspects of our lives. Worldwide, around 36 million people are blind and 217 million have a moderate to severe visual impairment. This portion of the population may use and encounter emoji, yet it is unclear what accessibility challenges emoji introduce. We first conducted an online survey with 58 visually impaired participants to understand how they use and encounter emoji online, and the challenges they experience. We then conducted 11 interviews with screen reader users to understand more about the challenges reported in our survey findings. Our interview findings demonstrate that technology is both an enabler and a barrier, emoji descriptors can hinder communication, and therefore the use of emoji impacts social interaction. Using our findings from both studies, we propose best practice when using emoji and recommendations to improve the future accessibility of emoji for visually impaired people.
\end{abstract}

\section{Author Keywords}

Emoji; CMC; Accessibility; Visual Impairments.

\section{CCS Concepts \\ -Human-centered computing $\rightarrow$ Accessibility;}

\section{INTRODUCTION}

There are currently over 3,000 emoji in the Unicode standard [27], and emoji are widely adopted in daily communication. In particular, emoji are prominent across social media: On Twitter over 25.4 billion tweets contain emoji (emojitracker.com), 5 billion emoji are sent daily on Facebook messenger [18], and in 2015 half of the comments and captions on Instagram contained emoji [19].

Emoji are also prevalent across many other aspects of society. Companies and marketers engage with audiences using emoji, some even paying up to 1M USD for custom emoji hashtags on Twitter [21]. In 2015, the 'Face With Tears of Joy' emoji was selected as word of the year [60] and Domino's allowed customers to text or tweet the 'Slice of Pizza' emoji fo to place an order (anyware.dominos.com). Emoji are evident in educational settings (e.g., learning management systems [12]),

Permission to make digital or hard copies of all or part of this work for personal or classroom use is granted without fee provided that copies are not made or distributed for profit or commercial advantage and that copies bear this notice and the full citation on the first page. Copyrights for components of this work owned by others than ACM must be honored. Abstracting with credit is permitted. To copy otherwise, or republish, to post on servers or to redistribute to lists, requires prior specific permission and/or a fee. Request permissions from permissions@acm.org.

CHI '20, April 25-30, 2020, Honolulu, HI, USA.

(C) 2020 Copyright is held by the owner/author(s). Publication rights licensed to ACM. ACM ISBN 978-1-4503-6708-0/20/04 \$15.00.

http://dx.doi.org/10.1145/10.1145/3313831.3376267 and are used by politicians and government bodies $[36,55]$, travel companies [54], media outlets, and public figures (e.g., singer Katy Perry who has one of the largest Twitter followings [51]). Emoji have even been discussed within official court transcripts [35], and resulted in convictions [23].

People interpret emoji differently, and emoji design variations across different platforms (e.g., iOS vs Android) can exacerbate misunderstandings [45, 64]. Furthermore, emoji are often used beyond their original intended meaning, which adds another layer of complexity to disambiguating the intended use of an emoji $[64,74]$. Prior research on emoji has largely focused on those with typical vision. However, it is estimated that 36 million people worldwide are blind and 217 million have a moderate to severe visual impairment [73]. Prior work highlighted challenges visually impaired people face when using technology [7] and social media [22, 49]. However, it is not clear what accessibility challenges occur with emoji.

The popularity of emoji means that any inaccessibility could lead to social exclusion, leading to a reduced quality of life. We surveyed 58 visually impaired people to understand the context around how they use and encounter emoji. Our survey findings highlighted challenges in searching for emoji, emoji design, misunderstanding and use in context, and the use of technology. We identified that emoji introduce more severe challenges for screen reader users. Therefore, we conducted 11 semi-structured interviews with visually impaired screen reader users to understand more about their experiences with emoji. Our thematic analysis demonstrated that technology was both an enabler and a barrier, emoji descriptors can hinder communication and use of emoji impacts on social interaction.

Using the findings from our two studies, we introduce best practice for using emoji with the aim of reducing accessibility challenges described by participants. We also propose recommendations to platform developers, social media companies, and the Unicode Consortium to address technical challenges that emoji pose to users of assistive technology.

Paper Contributions: We introduce three contributions: 1) Findings from an online survey with 58 visually impaired participants that report challenges experienced when using and encountering emoji online, which validate and extend anecdotal online discussion of emoji inaccessibility. 2) A thematic analysis of 11 interviews with screen reader users that describes how emoji introduce new barriers when accessing textual content. 3) We collate our findings into Best Practices and Technical Recommendations to improve the future accessibility of emoji for visually impaired people. 


\section{BACKGROUND AND RELATED WORK}

Computer-mediated communication (CMC), is communication between people that is facilitated by computers, such as email or social media [32]. Unfortunately, CMC removes important non-verbal cues from in-person interactions [33, 37, 47]. To address this, people used emoticons [15], which are ASCII characters depicting emotion (e.g., using a semi-colon and right parenthesis :) to show a smiley face). Use of emoticons can improve conversation [26] and message intent [40, 70], but have largely been replaced by emoji.

Emoji are a standardised set of unicode characters with visual representations of emotion, expressions, and objects [28]. As with any language, people have their own understanding and approach to using emoji. Emoji are useful for clarifying or enhancing message intent [14]. Emoji use also extends to symbolising private jokes, pictorial stories, and maintaining or showing interest in relationships [14, 56, 64, 74, 77]. However, emoji can be misunderstood due to variations in how their design is interpreted [44, 64]. Further miscommunication occurs because mobile platforms have their own designs (e.g., Apple iOS vs Google Android) [64]. The unicode characters for each emoji are constant across all platforms, yet the artwork varies. For example, the 'Face With Hand Over Mouth' emoji has the code point $U+1 F 92 D$ but can visually change (as shown in Figure 1). Knowledge about this rendering difference is not universal [46]. Research has shown there is a cultural gap with emoji design and user understanding or perception [34, 41], and personalities can also factor into how emoji are used [39].

Furthermore, searching for an emoji to use can also be difficult [53]. There has been some effort towards improving emoji input methods. In particular, Pohl et al. [52] introduced an emoji similarity model that can be used to improve emoji keyboard layouts. Auto-selection of emoji based on input is an alternative solution, with some work looking at improving the accuracy of emoji prediction [76].

\section{Visual Impairment and CMC Usage}

There are many challenges that visually impaired people (e.g., blind, low vision, impaired colour vision) face daily. This includes identifying product brands or names of objects, using technology, accessing digital services such as websites, and accessing equal social opportunities [7, 8, 13, 63, 72].

Social media remains one of the most popular ways in which we stay connected and heavily feature emoji [18, 19, 21, 25]. An analysis of 50,000 visually impaired Facebook users indicated that their engagement with Facebook was on par with the general population [75], highlighting it is imperative that accessibility is a key goal of social media. Morris et al. [49] found that as content on Twitter has become more visual, blind users were less likely to be able to participate. Twitter now allows users to enable image descriptions, however only $0.1 \%$ of images were found to include one [22]. Furthermore, Twitter users who have enabled image descriptions only use it on $\sim 50 \%$ of their images [22]. Alt text (alternative text) improves the accessibility of online images for screen reader users, yet has remained relatively unchanged since its inception [48]. Morris et al. [48] address this by proposing several ways in which to improve the alt text experience when accessing visual content. It cannot be expected that the assistive technology used will resolve all potential problems, but that social media platforms must also make accommodations to improve accessibility [10]. Numerous accessibility challenges with social media have been identified. However, there is insufficient research on emoji communication challenges.

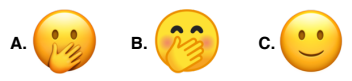

Figure 1. A.) 'Face With Hand Over Mouth' emoji on iOS and macOS. B.) 'Face With Hand Over Mouth' emoji on Android. C.) 'Slightly Smiling Face' emoji on iOS and macOS.

\section{Emoji Accessibility}

The Unicode Consortium is a non-profit organization that solicits proposals for new emoji. They also author and maintain a Common Locale Data Repository (CLDR) to store short character names for each emoji [3]. These descriptors are then used by screen readers to vocalise what the emoji visually represents. If we consider again the 'Face With Hand Over Mouth' emoji shown in Figure 1.A and 1.B, VoiceOver on macOS will read aloud the descriptor "face with hand over mouth with rosy cheeks". However, notice the descriptor is inconsistent. The blushing is 3D shading of the iOS design (see Figure 1.C). A screen reader user familiar with the descriptor may not realise the emoji does not match the descriptor.

There have been some attempts to improve emoji accessibility. For example, to address when emoji are sometimes not recognised by screen readers, one suggestion is to force the emoji to be recognised as an image and to give it an aria-label [71]. This technique can also be used to address the fact that emoji can have several meanings by providing the user with alternative descriptions [57]. Another approach to improve emoji accessibility for visually impaired people involved an investigation into tactile emoji [11]. Although the study found that visually impaired people could successfully identify tactile emoji to the represented emotion, it is not clear what the participant's current behaviour for using emoji were (if any) and what types of emoji accessibility challenges they encounter.

Individuals with low vision have expressed challenges of using emoji [69], such as distinguishing between them without the use of assistive technology, and difficulty inserting emoji into text without using dictation software. Solutions discussed include changing the skin colour of the 'thumbs up' emoji (using a skin tone modifier such as 'Fitzpatrick Type-4' ) to improve the visibility against certain backgrounds or requesting friends apply different colours to the 'thumbs up' emoji [69]. Work has explored designing new emoji specifically for visually impaired people [38], yet this is unlikely to solve problems caused by emoji use in the general population.

Sufficient attention on emoji accessibility is required. Therefore, we first conducted an online survey to understand how visually impaired people use and encounter emoji online.

\section{ONLINE QUESTIONNAIRE - METHOD}

We ran an online questionnaire with people who self-identified as visually impaired (e.g., Blind, impaired colour vision) to address three research questions: 1) How do visually impaired 
people use emoji? 2) Do visually impaired people encounter emoji? 3) What challenges do emoji and other forms of nontextual information present to visually impaired people?

\section{Materials}

The online questionnaire had 29 questions (12 closed-ended and 17 open-ended) across four sections. The first section contained eight questions and gathered basic demographic information: age, gender (we used an open text field in anticipation of a small sample size typical of accessibility research [58]), visual impairments, when visual impairments were acquired, visual acuity, assistive technology used to browse the internet and use messaging services, and message sending/posting frequency. The second section focused on using emoji and contained six questions. Participants were asked if they ever used emoji and if the response was "Yes", then the participants were asked how frequently they use emoji, to provide some examples of where and when emoji are used, to describe the reasons for using emoji, what the likelihood of using an emoji was within and outside of a work context. Participants who do not use emoji were asked to describe the reasons.

The third section focused on participants' experience of encountering emoji. Participants were asked if they had ever encountered emoji in content that someone else had written and if the response was "Yes", then the participants were asked how frequently they encounter emoji, to provide some examples of where and when emoji are encountered, what advantages there are to emoji being included within content, what disadvantages there are to emoji being included within content, and what the likelihood of encountering an emoji was within and outside of a work context. Participants who did not encounter emoji were asked to describe the reasons.

The final section explored the challenges emoji and other nontextual information present when used in content. Participants were asked how well they understood why other people use emoji in content, what challenges they experience when using emoji, what challenges they experience when encountering emoji, if they were aware emoji look visually different across platforms, and what other forms of non-textual information they encounter in content. Finally, participants were asked if they send other forms of non-textual information, and the advantages and disadvantages of each compared to emoji.

\section{Procedure}

Ethical approval was obtained from our IRB. We distributed the survey through social media (e.g., Facebook, Twitter), Reddit ( $r /$ samplesize, $r$ /blind, $r /$ colorblind, $r$ /glaucoma), university mailing lists, and by contacting charities and organisations (e.g., RNIB - rnib.org.uk, NFB - nfb.org). Admin permission was sought in all cases where we were outside of a group space. The participants were given an opportunity to enter into a prize draw for a \$50 USD (or equivalent) Amazon voucher. The survey was open from May 152019 for three weeks.

\section{Participants}

In total, 66 participants completed the questionnaire. Eight participants were removed from our analysis (five were under 18 years old, two did not respond to any questions, and one did not have a visual impairment). The remaining 58 participants (Male $=43$, Female $=12$, Undisclosed $=3$ ) were aged between $18-57$ years old $(\mathrm{M}=29.59, \mathrm{SD}=10.11)$.

We asked our participants about the number of visual impairments they have due to diversity in disability e.g. somebody could be colour blind from birth and later develop glaucoma. Data revealed: Single impairment (32 participants), two impairments (11), three impairments (5), four impairments (1), five impairments (2), and not given (7). The type of visual impairment varied greatly amongst the participants: Impaired Colour Vision (30 participants), nerve damage (9), blind (8), myopia (8), retina issues (5), eye development issues (4), nystagmus (3), macula issues (3), albinism (2), aniridia (2), astigmatism (2), low vision (2), photophobia (2). Finally, there was one participant for each of the following Axenfeld-Rieger syndrome, congenital cataracts, corneal edema, Leber congenital amaurosis, and punctate inner choroidopathy (PIC), and seven participants did not report details of their visual impairment. Our participants also reported on the development or occurrence of their visual impairments: Since birth (47 participants), $0-5$ years (3), 5-10 years (6), 10-15 years (2), 16+ years (3). There was one unclear response and two did not respond.

Participants were asked to rate their visual acuity using the textual descriptions proposed by the World Health Organisation (WHO) [20]: "None or Mild (equal to or better than 20/70)" (27 participants), "Moderate (worse than 20/70 and equal to or better than 20/200)" (9), "Severe (worse than 20/200 and equal to or better than 20/400)" (5), and "Blind (worse than 20/400)" (12). Four participants provided different responses: "colorblind, I don't know sorry", "normal with glasses", "with glasses, left acuity can be brought to None or Mild, besides blind spot which is 0 ; right acuity is blind", and "one eye is $20 / 40$ with correction. One eye is worse than 20/400 with correction". One participant did not respond to the question.

Our participants used a variety of assistive technologies when browsing the internet and using messaging services. We found colour filters (both digital and physical, such as tinted glasses) and identifiers were used by 18 participants. Overall, screen reader software was used by 17 participants: JAWS (6 participants), VoiceOver (5 participants), NVDA (3), and TalkBack (3). Other assistive technologies participants reported using were: Magnification (10), glasses/contact lenses (5), braille or braille displays (4), none (2), and not given (11).

To build an understanding of the participants behaviour we asked how often they send messages (e.g., text messages, email) and post messages online (e.g., Facebook posts, tweets). We found 49 participants send messages at least once a day and nine participants at least once a week. Finally, 27 participants reported they post messages online at least once a day, 19 participants at least once a week, four participants at least once a month, and eight participants less than once a month.

\section{Analysis}

We analysed our open-ended responses using open coding [67] based on an existing procedure [65]. We analysed each question independently using a four-step process: 1) Initial coding: The first author read all responses and generated initial codes 
with a data-driven approach. Codes were collated and collapsed into an initial codebook. 2) Evaluating codes: The first two authors independently coded $1 / 3$ of the responses (randomly-selected) using the initial codebook, agreeing to identify each 'mention' once. Codes and descriptions were refined by discussing disagreements. 3) Coding the full data set: The same authors separately re-coded all responses with the updated codebook and rules. 4) Defining themes: The same authors reviewed the final coding to identify similarities that allowed thematic grouping. We collated codes into themes and therefore did not calculate survey inter-rater reliability because codes were not the final outcome of our analysis [43].

\section{ONLINE QUESTIONNAIRE - FINDINGS}

We present our findings, using closed-response data and participant quotes, under three thematic sections: 1) Using Emoji, 2) Encountering Emoji and 3) Challenges of Emoji.

\section{Using Emoji}

We asked our participants if they have ever used an emoji (i.e. sent or posted online) and found that 56 participants had and only two participants had not. The reasons given for not using emoji, by the two participants, included: the size of emoji (1 participant), that they did not think about using emoji (1), and that using text-to-speech made it difficult (1). Participants who used emoji reported varying frequencies, as summarised in Table 1. Our subjective scale ranged from "Less than once a month" to "At least once per day" because further granularity is difficult for participants to accurately recall [61]. Overall, $55 \%$ of participants used emoji at least once per day and $74 \%$ of participants sent emoji at least once per week.

\begin{tabular}{cc}
\hline Frequency of Using Emoji & No. of Participants \\
\hline At Least Once a Day & 32 \\
At Least Once a Week & 11 \\
At Least Once a Month & 5 \\
Less than once a month & 7 \\
Not Given & 3 \\
\hline
\end{tabular}

Table 1. Frequency participants reported using emoji.

Overall, participants reported diverse examples of where and when they used emoji: private (35 participants) and public contexts (20), during conversations (9), for conveying emotion and reactions (9), within a work or productivity context (7), for fun or to add humour (7), for clarification in messages (4), when at home (1) or travelling (1), and as a result of systems automatically suggesting or inserting emoji (1). Two participants mentioned not sending or using emoji and five participants did not answer or provided a non-useful response.

\section{Reasons for Using Emoji}

Participants described many reasons for why they use emoji. Participants mentioned the benefit of clarifying or enhancing message content (46 participants), the fun aspect or adding humour (26), the speed or ease of making a statement or replying to a message (10), the ability for unique usage such as assigning alternative meaning to emoji (3), using emoji with friends and family (1), utilising the less formal nature of emoji (1), using emoji for work or with colleagues (1), the social pressure to fit in with others (1), and as a result of systems automatically suggesting or inserting emoji (1). Two participants mentioned not sending or using emoji and three participants did not answer the question or provided a non-useful response. Many participants mentioned the use of emoji to enhance and clarify content within communication, for example to avoid “...words being taken the wrong way." (P27). Some participants conveyed clear enjoyment of using emoji, noting that emoji were "cute" (P66) and that their use "adds something fun to messages" (P10). P51 specifically commented that they use emoji to be "...perceived as someone who is able to embrace sighted culture". We also asked participants to report what the likelihood was of them using emoji within and outside of a work context (as summarised in Table 2). Overall, participants are more likely to use emoji outside of their work environment.

\begin{tabular}{ccc}
\hline $\begin{array}{c}\text { Likelihood of } \\
\text { Using Emoji }\end{array}$ & $\begin{array}{c}\text { Within } \\
\text { Work Context }\end{array}$ & $\begin{array}{c}\text { Outside } \\
\text { Work Context }\end{array}$ \\
\hline Always & 0 & 5 \\
Likely & 6 & 28 \\
Neutral & 4 & 11 \\
Unlikely & 28 & 11 \\
Never & 17 & 1 \\
Not Given & 3 & 2 \\
\hline
\end{tabular}

Table 2. Participants' reported likelihood of using an emoji within and outside a work context (Neutral is neither likely nor unlikely).

\section{Encountering Emoji}

We asked our participants if they have ever encountered (i.e., read) an emoji in text that someone else has written (e.g., in a message or tweet) and found 56 participants had, one participant had not, and one participant did not answer the question. Participants reported varying frequencies of encountering emoji as summarised in Table 3.

\begin{tabular}{cc}
\hline Frequency of Encountering Emoji & No. of Participants \\
\hline At Least Once a Day & 45 \\
At Least Once a Week & 8 \\
At Least Once a Month & 1 \\
Less than once a month & 2 \\
Not Given & 2 \\
\hline
\end{tabular}

Table 3. Frequency participants reported encountering emoji.

Overall, participants reported a variety of situations where they have encountered emoji: in private (43 participants) and public (32) contexts, with friends and family (14), at work or with colleagues (7), when emotion and reactions needed to be conveyed (7), at home (1), and during significant events or anniversaries (1). Three participants did not answer the question or provided a non-useful response.

To better understand the context around when participants encountered emoji, we asked them to report what the likelihood was of them encountering emoji within and outside of a work context (as summarised in Table 4). Overall, participants were more likely to encounter emoji outside of their work environment, as was also the case with using emoji. 


\begin{tabular}{ccc}
\hline $\begin{array}{c}\text { Likelihood of } \\
\text { Encountering Emoji }\end{array}$ & $\begin{array}{c}\text { Within } \\
\text { Work Context }\end{array}$ & $\begin{array}{c}\text { Outside } \\
\text { Work Context }\end{array}$ \\
\hline Always & 1 & 10 \\
Likely & 7 & 34 \\
Neutral & 5 & 7 \\
Unlikely & 33 & 4 \\
Never & 9 & 0 \\
Not Given & 3 & 2
\end{tabular}

Table 4. Participants' reported likelihood of encountering emoji within and outside a work context (Neutral is neither likely nor unlikely).

\section{Advantages of Encountering Emoji}

Participants described many advantages of emoji being included within content, including: for clarification within messages (37 participants), increased speed or ease of making/replying to a statement (9), for fun (5), and because emoji are useful as a universal language (1). Nine participants mentioned negative aspects of emoji or statements about emoji offering no advantages with four participants not answering or providing non useful responses. As with using emoji, the most popular advantage was to clarify or enhance content within a message, such as described by P46: "It can convey things beyond words". Speed and ease of use were also mentioned, such as they "save time and convey [emotion] clearly" (P58), and emoji are "easier to express a feeling" (P6). One participant also discussed that the "empathy aspect is also valuable, particularly when people tell a story with emoji" (P55).

\section{Disadvantages of Encountering Emoji}

Participants outlined disadvantages to emoji being included within content. The responses included: emoji are informal or non-serious nature (15 participants), over reliance of emoji over real words (12), the risk of confusion and misunderstanding (12), technology challenges due to emoji (11), repeated auditory feedback when multiple emoji are used (6), visual clutter caused by multiple emoji within a message (5), finding an emoji to use (2) and the difficulty of perceiving emoji (1). Eight participants mentioned negative aspects of emoji or statements about emoji offering no advantages and three participants did not answer or provided non-useful responses.

\section{Challenges of Emoji}

Participants self-reported how well they understood why other people use certain emoji in their writing. We categorised participants' comments on their understanding into Excellent (13 participants), Good (22) and Poor (4). This was based on a broad understanding of participant comments and not as a judgement of their level of 'correctness'. Some participants gave specific examples of why they think others use emoji. These included: the ability to more expressive ( 9 participants), some emoji are used because they are easy to understand based on the look or surrounding context (8), to try and avoid a misunderstanding (7), for fun or to add a personal touch (5), when there is good knowledge of the person and their communication style (4), to keep messages concise (3), and because emoji are useful as a universal language (1). Nine participants did not answer the question or provided a nonuseful response and one participant's response was unclear.

\section{Challenges of Using Emoji}

Participants reported the challenges they experienced when using emoji and included: searching for an emoji to use (26 participants), challenges related to emoji visual design (13), resulting in confusion and misunderstanding (8), the technology challenge that emoji introduce (8), issues due to emoji size (4), limited access to appropriate emoji for the context (4), and the situation or message not agreeing with the emoji (3). Overall, 10 participants reported no challenges and eight did not answer the question or provided a non-useful response.

Searching for Emoji: Overall, $45 \%$ of participants reported challenges when searching for an emoji to use. In particular, this was related to finding an emoji that fitted what they were trying to convey in written text such as $\mathrm{P} 4$ who said that it was "...hard to find the one [to] represent what I mean".

Technology: Participants mentioned that they sometimes experienced issues related to selecting emoji. For example:

P44: "Sometimes if you miss click an emoji it might get weird. Like if you send a heart [ $\mathrm{Q}]$ to someone you'd never send a heart to and then have to explain it was an error, which also might be weird."

Participants also reported that touch screen settings were challenging: "not being able to find them quickly or $3 D$ touch being a little too twitchy...so getting the wrong ones" (P50).

Visual Design: Emoji design also caused challenges for $22 \%$ of the participants. For participants who had some residual vision, this was often related to the use of colour such as P6 who described that "the colors of the heart [emoji] can be too similar.". For blind participants, differences between design of the visual emoji and the description were challenging:

P28: "Some emoji [are] useless or just have a bad design (I was told the 'pray' emoji [\$] is actually a 'high five')."

Misunderstanding: This relates to the use of visual representations of things that blind users had not experienced. This sometimes made it difficult to select an emoji.

P38: “...I entered the word 'happy', and it suggested many faces, which were all described to me; however, as I have never had vision, I was unable to know which face was the most appropriate for my situation."

Emoji Size: For participants with residual vision, they described trying to identify specific emoji, but finding it difficult when many are presented on small mobile device screens: "I only use a select few as most expressions or objects are too small to identify" (P59).

Limited Access: Some participants noted that there are "limited images to choose from" (P19). This may be because the emoji are displayed across multiple screens, which can make it a challenge to find a specific one.

P03: "So many emoji without a search bar for example. It leaves me going back to endless pages of obsolete ones."

Context: For some participants, context was a challenge when selecting and sending an emoji. Participants noted specific 

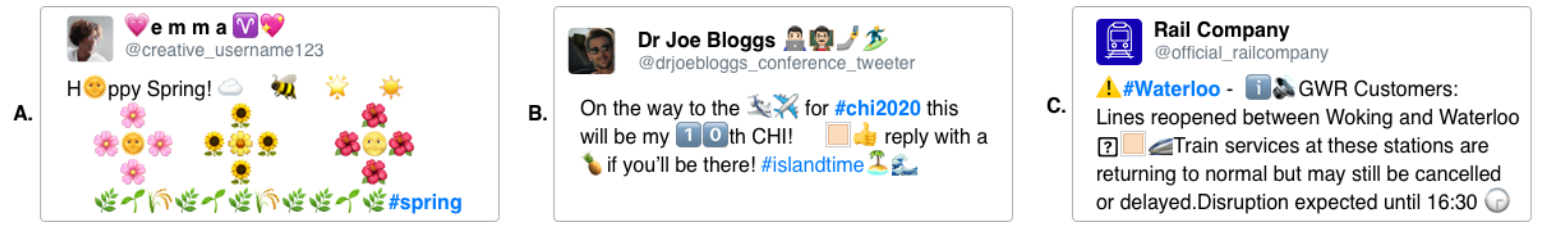

Figure 2. Three recreations of real tweets illustrating problems reported by our survey and interview participants. A) A tweet where the author has used emoji to draw a picture of three large flowers. B) A tweet sent by a conference attendee. Rather than using the word airport they use two plane emoji and asks fellow attendees to reply with an emoji. C) A tweet sent by a rail company containing important information about rail delays.

emoji, e.g. the 'peach' emoji, which has an alternate meaning within popular culture: "One example is the peach emoji [-1], I have never used it myself, but only recently became aware that it's generally accepted to be a butt." (P9). See Figure 2.B for another example of context issues.

\section{Challenges of Encountering Emoji}

We asked participants if they were aware that emoji looked visually different across platforms. Forty-eight participants were aware, eight participants did not know, and four participants provided no response. Participants noted "There should be standardization." (P19) and gave examples of experiences:

P43: "I had no clue about this... [my sister] told me that on iOS, emojis look more high-quality and visually appealing”.

In addition, all participants were asked what challenges they experienced when reading emoji. Challenges given were the risk of confusion and misunderstanding (14 participants), the technology challenge that come with emoji (13), issues with emoji size (10), understanding why an emoji was used in a particular context (9), challenges related to emoji design (7), and challenges related to people misusing emoji (7). Fourteen participants reported no challenges and five participants did not answer the question or provided a non-useful response.

Misunderstanding: This was the most common challenge reported and was related to subjective understanding of intent:

P43: "Sometimes when [people] send an emoji to a blind person, the emoji is meant for a different thing and more often than not, does not sound the same as it might look."

Participant level of exposure was not a factor in the misunderstandings reported: 10/14 participants who reported the biggest challenge to be risk of confusion and misunderstanding also encountered emoji at least once per day.

Technology: Participants commented that there was a lot of variation in emoji between different devices and applications, and that some emoji may not be supported on different platforms or devices such as screen readers:

P19: "The facial expressions vary from app to app, which means that a super excited face on one app might be an an excited yet angry face on another."

Context: Participants discussed the importance of context, with some explaining how they were not always able to determine the intent of a message from the emoji descriptors:

P32: "The alt text may not match the context that the user is trying to provide making the overall intent of the message confusing to a screen reader user."
This was more problematic when emoji were used as decoration (e.g., the pictorial drawing shown in Figure 2.A), and the descriptors were not related to the surrounding semantics.

Visual Design: For participants with residual vision, the small size of the emoji contributed to challenges in receiving emoji such as P7 described: "Sometimes the [emoji] face is too small to understand.". The visual features of the emoji can also contribute to this challenge. P10 described being "unable to distinguish different colors of the emojis". P20 reported having "difficulty seeing the expression on the emoji.".

Misuse: Finally, participants noted situations where they considered emoji to be misused. For example, P53 reported that "Too many [emoji] used in one message make it laborious to read ..." and this is challenging to interpret when using a screen reader or other assistive technology. An example of overuse can be seen in Figure 2.A and 2.B.

\section{Sending Other Non-Textual Information}

We asked participants if they send other forms of non-textual information, and to state any advantages and disadvantages of each one compared to emoji. The non-textual information noted by participants was emoticons (16 participants), GIFs (14), images (10), audio (6), video (5), memes (2), ASCII art (2), stickers (2), and 27 participants did not provide a response. Many participants described emoticons as other methods of conveying non-textual information, and did so positively:

P51: "Emoticons are easier to type than emoji for me, especially when typing in Braille and not at a computer."

Finally, participants described how other non-textual information allowed them to convey more expression and detail:

P66: "Gifs, audio, and video allow for a more full context of what someone is saying or feeling than an emoji or emoticon can. Emojis and emoticons are easier to use though."

\section{Summary of Questionnaire Findings}

Our findings highlighted numerous challenges faced by visually impaired people when using and encountering emoji. This included searching for emoji to use, emoji design, misunderstanding and use in context, and the use of technology. The negative impact emoji had on users of assistive technology was considerable, especially for screen reader users. These users are typically reliant on the emoji descriptor (e.g., 'Face with Tears of Joy' emoji ), which can make emoji challenging to understand, as the descriptor may not match the intended use. To understand more about the challenges screen reader users encounter when using and encountering emoji online, we conducted one-to-one interviews with screen reader users. 


\section{SCREEN READER USER INTERVIEWS - METHOD}

We had two main research questions guiding our interviews: 1) What challenges are experienced by screen reader users when sending emoji? and 2) What challenges are experienced by screen reader users when perceiving and understanding emoji?

\section{Procedure}

After obtaining ethical approval from our IRB, we conducted semi-structured one-to-one interviews using online messaging tools. We did this for two reasons: First, interviewing over a messaging service would allow participants to share emoji if they wanted to provide examples; Second, there would be more convenience for participants (e.g., not having to go somewhere private to talk over the phone). We selected the tool in collaboration with each participant to ensure accessibility (since our participants were users of screen readers). The third author conducted all interviews. A pilot interview was held with the second author prior to beginning the study. Participants were recruited using the same methods used for the survey, and were reimbursed for their time with an Amazon voucher equivalent to \$20 USD. The mean interview time was 62 minutes ( $\max 70$ minutes, min 48 minutes). Participants completed a pre-questionnaire to gather demographic data (see supplementary material) that was anonymised for analysis.

\section{Participants}

We interviewed 11 participants (Male $=8$; Female $=2$; Agen$\operatorname{der}=1)$, aged between $18-37$ years old $(M=28, S D=6.15)$. Of these 11 participants, 10 believed that their visual impairment impacted their use of emoji. Participants were asked to rate their visual acuity using the textual descriptions proposed by the World Health Organisation (WHO) [20]: "Severe (worse than 20/200 and equal to or better than 20/400" (1 participant), and "Blind (worse than 20/400)" (9 participants). One participant provided a different response: "Full blindness with zero light perception but physical eyes still remain".

We asked participants about the visual impairment(s) they have: Single impairment (8 participants), and two impairments (3). The type of visual impairment varied greatly amongst the participants: Blind (8 participants), Low Vision (1), Retina Issues (2), Nerve Damage (1), Axenfeld-Rieger syndrome (1). Our participants also reported on the development or occurrence of their visual impairments: Since birth (4 participants), 0-5 years (4), 5-10 years (0), 10-15 years (1), 16+ years $(2)$.

All participants were self-reported screen reader users and used a variety of software: Voiceover (10 participants), NVDA (5), JAWS (3), and Talkback (1). All participants reported sending messages (e.g., Facebook messages, SMS) at least once per day. Posting messages online (e.g. discussion forums, Facebook posts) was more varied with participants reporting this action at least once per day (7 participants), once per week (1), once per month (1) and less than once per month (2).

We asked participants how often they sent emoji. This was an open field so more details could be provided. All participants had sent emoji; responses ranged from "every day" to "hardly ever". Two participants also mentioned using emoticons more often than emoji. We also asked participants how often they receive emoji. Responses were less varied, with participants stating "almost every day" as a minimum frequency, with most participants stating "daily" or "all of the time".

\section{Analysis}

We analysed our interview transcripts using thematic analysis [9]. The first and third author read all responses and took note of initial codes. Codes were generated using a data-driven approach then collated and collapsed. The same authors then reviewed the final coding and identified similarities to allow thematic grouping by creating an initial thematic map. Our final thematic map is shown in Figure 3. We did not conduct inter-rater reliability because it is not part of Braun and Clarke's checklist for good thematic analysis [9], and there is debate if it is suitable for this type of analysis [5, 42, 24].

\section{SCREEN READER USER INTERVIEWS - FINDINGS}

Through our thematic analysis, we identified three themes: 1) Technology is both an enabler and a barrier, 2) Emoji descriptors can hinder communications, and 3) Use of emoji impacts social interaction. We now explore, and scaffold the narrative of each theme in detail using quotes from participants.

\section{Technology is Both an Enabler and a Barrier}

For visually impaired people, screen readers are crucial for accessing visual content, however our interview participants described that screen readers could be a barrier to emoji accessibility. In particular, participants highlighted a range of challenges related to Searching and Selection, Output from Technology, Up-to-Date and Knowledge About Technology.

Searching and Selection: All of our participants found searching and selection of emoji to be challenging. There are several elements, such as the organisation of emoji lists, the available mechanisms for finding emoji (e.g., keyword searching), and knowing what is available. In particular, the increasing number of emoji that exist is further highlighting this challenge.

P2: “...finding the right one to send. I either don't know whether it exists or what it is, or where to find it. Sighted people just glance at a screen and can find them pretty quickly, while we have to go through all of them."

In relation to the large numbers of emoji, $\mathrm{P} 3$ recommended that some emoji are grouped together to simplify searching, e.g. "It would help to have an option to change the skin tone instead of having them all there". Some participants outlined alternative solutions for when they are unable to find a specific emoji:

P9: "I have spent a lot of time looking for emoji that I know exist but cannot find...I have sometimes found an old instance of the emoji in a previous conversation and copied it."

Output from Technology: Our participants noted that it could be challenging to identify emoji within output, especially where emoji are disabled or incompatible. Different screen reading technologies may describe emoji in different ways.

P4: "JAWS describes [2] as 'face with look of triumph' while Voiceover describes it as 'huffing with anger face' and according to my sighted brother, Voiceover's description is more accurate." 
Figure 3. Final thematic map of three main themes and their sub-themes: 1) Technology is both an enabler and a barrier, 2) Emoji descriptions can hinder communications, and 3 ) Use of emoji impacts social interaction.

Participants also noted that reading emoji can lengthen the output from screen readers, which can be inconvenient.

P7: "Whenever someone sends a string of emojis as a joke, it is annoying to have to hear them all strung together."

Up-to-Date: Our participants highlighted that their assistive technologies were not always up to date and this meant that emoji were not always fully supported. For example, new emoji were not added, or that there was a reliance on third party applications such as screen reader libraries or soft keyboards.

P1: "The [NVDA] plugin is very out of date and supports 100 mixed emoji and emoticons, [but] the built-in dictionary supports $\sim 3000$ but that's mixed emoji and symbols..."

Knowledge About Technology: Our participants highlighted that different screen readers were available and they needed to be technically aware in order to access different features that could help them. The level of competence varied between participants, e.g., one participant was aware of the punctuation settings on NVDA, which could avoid repeated emoji being read out in their entirety, but others were not. Indeed, some participants recommended such a feature to us as a solution.

\section{Emoji Descriptors Can Hinder Communications}

On a screen reader, emoji descriptors are output as speech or braille and describe the visual design of an emoji. However, the descriptor does not always accurately describe the visual design, which can lead to challenges when using emoji:

P6: "Emoji is something fun for sighted texters...but for me it's just an extra string of words. ...like the grinning face emoji [:-)]; it looks fun and cute when you look at it, but Voiceover describes it as 'grinning face with clenched teeth emoji' which sounds more like a grimace than a big smile)."

For complex emoji, the descriptors can also be verbose, which makes communication with a screen reader cumbersome. P7 added there was a "user education issue" and that "if sighted users knew what the [descriptor] was, it may help".

\section{Use of Emoji Impacts Social Interaction}

Our participants described how using emoji in conversations could lead to communication breakdown and social exclusion. Our participants highlighted challenges related to Poor Use in Context, Conversational Flow and Cultural Differences.

Poor Use in Context: Our participants highlighted that emoji used in different contexts can lead to specific challenges. Decorative emoji, e.g. emoji in usernames on social media, caused challenges as many decorative emoji could be announced by a screen reader. An example of this is shown in Figure 2.A.

P7: "Try listening to 'cat with heart shaped eyes fireworks sparkles watermelon kissing face flag of Andorra' a few times in a row and you get the frustration."
It is possible to avoid screen readers announcing emoji in certain contexts, e.g. in usernames, as reported by one participant, but this was reliant on an unofficial plugin. Descriptors often did not match the intended purpose, e.g. emoji were selected based on their visual representation, leading to misunderstanding. See Figure 2.C for an example.

P8: "Email subjects have emoji now; Ebay put a [a] to show your order has been sent. For a long time, I [was] puzzled as to why they'd write the word 'truck' there."

Conversation Flow: Our participants discussed how emoji enhance conversations, such as enabling quick replies. Misunderstandings could change the conversation tone, and were more likely when unfamiliar emoji were used. Some participants reported ignoring a conversation when emoji were used extensively, so both context and content was missed. For example, P2 did not know about an important test being rescheduled because the information was lost within emoji:

P2: "in the middle of [multiple heart emoji], someone posted something else, which was also important. I didn't pay attention [to] the wave of hearts, so I didn't know".

Cultural Differences: Our participants highlighted differences between sighted and non-sighted culture and a desire for social inclusion. Participants described aiming to engage with sighted popular culture using emoji, but often relied on emoticons as they were platform independent and more easily understood by their visually impaired peers. Participants who had been blind since birth commented that the link between visual representation and intent can be challenging.

P8: "Imagine you're totally blind, you'd never seen the gesture. So that has to be learned. You can write 'no' or 'that's bad' etc. Choosing to send a pictorial representation of a negative feeling may pose more of a challenge."

In addition, P6 discussed feeling excluded from society:

P6: "It's a bit frustrating and depressing. I don't follow many people and it's sad to suddenly be shut out of content or a conversation solely because of a text decoration trend."

\section{Summary of Interview Findings}

Our findings reveal emoji challenges experienced by screen reader users. We found that screen reader technology is both an enabler and a barrier. Emoji descriptors also introduce problems and they can result in misunderstandings, and therefore the use of emoji has a significant impact on social interaction.

\section{BEST PRACTICES \& RECOMMENDATIONS}

Emoji are part of most social platforms and modern communication. Our findings describe why people may choose not to use emoji, but encountering emoji is something that users have little influence over. Emoji accessibility is an area not fully understood and is having a detrimental impact on the 
social inclusion of visually impaired users. To address this, we use our findings to introduce emoji use best practices and recommendations for future emoji development.

\section{Best Practice When Using Emoji}

People who use emoji should consider the sender, the reader or recipient, and the platform that is being used to both send and receive the emoji. Understanding the needs of the end user should be a primary consideration.

1) Number of Emoji: Repeated emoji can cause considerable annoyance and frustration. If you wish to say that something is amusing and are using the 'Face with Tears of Joy' emoji, consider that each time you add that emoji may increase the number of times the descriptor is read out:

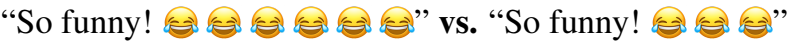

Therefore, consider how many emoji are necessary in content.

2) Placement of Emoji: Consider that a sentence with emoji will be read by a screen reader as if the emoji were text. This is especially important because the descriptors may not match with your expectations (e.g., + is read as "sun", not "sunny").

$$
\text { "It is today." vs. "It is sunny today }
$$

Emoji in usernames (such as on Twitter) should be avoided. At a minimum, consider placing emoji at the end of your username, which means that screen reader users can skip past the emoji once they understand who is posting. Therefore, consider placing decorative emoji at the end of content, or at a minimum at the end of each line of content.

3) Purpose of Emoji: The descriptors for emoji are not always a clear indication of the visual design or the emotive intent. Such information should also be represented in the surrounding text. Therefore, consider that emoji (or the descriptor) should not be used to convey critical information in content.

4) Consideration of Reader: An understanding of how different users can perceive emoji is important. When your emoji has a greater reach (such as on a public platform), there is a greater chance that the emoji will be encountered by visually impaired people. Therefore, consider the wider context before using emoji and ensure that the accessibility of your content is evaluated before sending or sharing.

\section{Recommendations for Future Emoji Releases}

Our recommendations discuss technical issues that need to be considered by vendors and organisations to further improve the accessibility of emoji prior to future emoji standard releases.

1) Emoji Descriptors: Discrepancies between the visual design of emoji and their descriptors can cause confusion and misunderstanding. We recommend that descriptors and visual designs are approved for consistency by the Unicode consortium. Progressive detail [48] could provide additional context for screen reader users and allow screen reader users to make more informed use of emoji. Vendors and assistive technology designers should consider implementing progressive detail.

The presentation of duplicate emoji also varies between screen readers, with some allowing users to reduce repetition, e.g., "three 'Face with Tears of Joy' emoji". As emoji become more widely used, this setting should be implemented on all screen readers and made more prominent to users.

Additions to the emoji standard could also increase issues caused by descriptors. For instance, the Unicode Committee is reviewing whether to add colour modifiers to Unicode Emoji V13.0 [30]. This mechanism would use the emoji colour characters (e.g., seven coloured square characters at U+1F7E6 $-\mathrm{U}+1 \mathrm{~F} 7 \mathrm{~EB}$ ) to allow additional emoji representations such as a glass of 'White Wine' ('Wine Glass' + 'White Square' ). However, these coloured emoji would be represented by two emoji, so the underlying emoji descriptors would be "Wine Glass, White Square" and not 'White Wine'.

2) Unsupported Emoji: Unsupported emoji occur when one platform, e.g. Apple, releases new emoji faster than others, e.g. Android. New emoji are then encountered on devices with an outdated emoji standard. The new emoji are not rendered correctly, nor do they have an updated list of the emoji descriptors as a fallback. We recommend that new emoji are embargoed until a specific date after a standard has been approved.

Further challenges due to unsupported emoji would arise if Unicode approve QID Emoji Tag Sequences or QID emoji in Emoji Standard V13.0 [30]. QID emoji would allow for communities and companies to use this mechanism to put together their own sets of emoji. The character of each of these emoji is established by reference to a Wikidata QID (unique identifier used by Wikidata). For instance, the 'Sauropod' emoji $h+$ Q14384 (Triceratops QID - wikidata.org/wiki/Q14384) could result in a 'Triceratops' emoji , but only if a valid visual design was available. However, if there was no visual design available, the fallback for screen readers discussed by Unicode [30], would be to indicate that there was an emoji present and not provide any indication to what that emoji represented. We recommend that Unicode think carefully about the accessibility implications of allowing external organisations and companies to add additional non-standardised emoji.

3) Platform Visual Differences: Platform differences cause misunderstandings between users, and these are greater when a user has a visual impairment. The Unicode design guidelines [29] state that platform differences are possible yet a design that varies significantly from other vendors' representations may cause interoperability problems. We recommend that visual designs by each platform should be approved by Unicode to ensure adherence to the emoji descriptor.

4) Facilitating Emoji Use: Emoji selection currently requires a visual search. Some work has been completed toward alternative emoji selection [53,17], but none are specifically designed for visually impaired people. Vendors should develop alternative emoji input methods. Employing co-design methods with visually impaired people is imperative in the design and evaluation of new solutions. For example, custom gesture-based input could be one direction $[4,6,50]$.

5) Diversity in Design Process: Our participants did not discuss how emoji designs were/were not representative of people with different abilities. However, it is unclear if users are represented within Unicode [68], or are involved in the development 
of new standards. Inclusive design processes are established practice for the development of accessible technology [31]. We recommend that an accessibility sub-committee is formed to include people with a diverse range of abilities, which will lead to more representative emoji in terms of both design and access needs. There has also been a call to democratise emoji design through more public engagement [62], which could be an additional step towards improving emoji accessibility.

\section{DISCUSSION \\ Summary of Contributions}

1) Questionnaire data on emoji usage from 58 visually impaired participants: Our findings demonstrate that emoji introduce a number of challenges for visually impaired people when using, and encountering emoji online. This includes searching for emoji to send within content, emoji design, misunderstanding emoji intent and their use in context, and the impact emoji has on assistive technology. Our findings extend prior work in this area [14, 44, 45, 64, 74], and confirm anecdotal online discussion regarding how emoji can introduce accessibility challenges for visually impaired people, which are more severe for screen reader users.

2) Thematic analysis of 11 interviews with screen reader users: Our interview findings with screen reader users demonstrate that screen reading technologies are both an enabler and a barrier, emoji descriptors can hinder communication, and the use of emoji impacts social interaction. These findings show that the use of emoji within textual content has reintroduced accessibility barriers to these users, which had previously been levelled with the use of screen reading technologies.

3) Best practices and technical recommendations: We used our findings to inform best practices when using emoji. These should be considered by all users who post content that could be read by visually impaired people, and is important for public bodies and companies who could be in breach of equality laws $[1,2]$. We introduce recommendations to improve the accessibility of emoji for visually impaired people. These should be considered by all organisations involved in emoji, such as the Unicode Consortium, platform developers (e.g., Apple, Google), social media companies (e.g., Twitter) and developers of screen readers and other assistive technology.

\section{Limitations}

Fifty-eight visually impaired people completed our survey, and while accessibility research recruitment is a challenge [59], our results may not generalise to the wider population. We focused on visual impairment challenges, yet some challenges may be from cultural differences $[34,41]$. However, our survey was in English and thus the majority of our participants may be from English speaking countries, potentially limiting cultural influences of misunderstanding. Further exploring cultural differences and emoji accessibility would be interesting.

There is a limitation due to respondents' ability to accurately recall their experiences of using emoji. However, the majority of our participants used (58\%) and encountered (77\%) emoji daily, so the impact of this on our findings may be minimal. Our survey participants reported a wide variety of visual impairments. However, only $\sim 34 \%$ of participants described their visual acuity to be worse than "None or Mild" (i.e., worse than 20/70). This could be explained by the number of visual impairments reported by participants, along with the number of participants with colour vision deficiency within our sample. We did not ask about participants' social media usage since emoji are found in many different platforms of communication. Our findings support this reasoning since emoji issues raised included but were not limited to social media.

We had 11 participants take part in our interviews. Due to this sample size our results may not generalise to all screen reader users. We were unable to obtain a gender balanced group, yet it is well known that recruiting participants for accessibility research is a challenge [59]. We also cannot determine who may have took part in the prior survey since it was kept anonymous. Participants took part in our interviews over messaging tools using assistive technology. This could have limited participants' expression versus an in-person interview, although this is unlikely to significantly affect the quality of the data [16]. There may also be a self-selection bias for participants who were more confident using assistive tech.

\section{Generalisations \& Future Work}

We focused on understanding challenges faced by visually impaired people due to emoji. Some of these challenges may also apply to users with typical vision as a result of situationallyinduced impairments and disabilities (SIIDs) [66]. For example, SIIDs caused by screen glare or low screen brightness may result in challenges with selecting emoji, due to their small dimensions. In addition, this is likely to be an issue on smartwatches and on augmented reality screens. The use of Voice Assistants (VA), such as Amazon Alexa (alexa.amazon.com) and car-based support such as CarPlay (apple.com/carplay) are now commonplace. VAs announce content, and thus need to read emoji descriptors in similar ways to screen readers. This may introduce similar challenges, such as misunderstandings of emotive intent, for users in different contexts.

\section{CONCLUSION}

Emoji are prevalent within communication, however the accessibility challenges they introduce are not well understood. We conducted an online questionnaire with 58 visually impaired participants to explore their experiences. We found that emoji introduce several specific challenges for screen reader users, notably around social communication. We then conducted 11 interviews with screen-reader users to further understand the challenges they face when encountering emoji. Participants raised issues that ultimately resulted in social exclusion. Considering the challenges we have identified, the quality of communication will continue to diminish as new and more complex emoji are released. To address this, people should consider our best practices when using emoji, and vendors and organisations should consider our recommendations when determining the future direction of emoji.

\section{ACKNOWLEDGEMENTS}

We thank our participants for taking part in this research, and to Erin Brady for supporting early stages of this work. This research was awarded funding via the Bournemouth University ACORN (Acceleration Of Research \& Networking) Fund. 


\section{REFERENCES}

[1] 2000. Section 508. Government of the United States of America. access-board.gov/guidelines-andstandards/communications-and-it/about-the-section508-standards/section-508-standards Accessed: 2019-09-17.

[2] 2010. The Equality Act. London: The Stationary Office. legislation.gov.uk/ukpga/2010/15/contents Accessed: 2019-09-17.

[3] 2019. Unicode ${ }^{\circledR}$ Emoji Chart Format. https://unicode.org/emoji/format.html\#col-name. (2019). Accessed: 2019-03-22.

[4] Jessalyn Alvina, Carla F. Griggio, Xiaojun Bi, and Wendy E. Mackay. 2017. CommandBoard: Creating a General-Purpose Command Gesture Input Space for Soft Keyboard. In Proceedings of the 30th Annual ACM Symposium on User Interface Software and Technology (UIST '17). Association for Computing Machinery, New York, NY, USA, 17-28. DOI :

http://dx.doi.org/10.1145/3126594.3126639

[5] David Armstrong, Ann Gosling, John Weinman, and Theresa Marteau. 1997. The place of inter-rater reliability in qualitative research: an empirical study. Sociology 31, 3 (1997), 597-606. DOI : http://dx.doi.org/10.1177/0038038597031003015

[6] Shiri Azenkot, Cynthia L. Bennett, and Richard E. Ladner. 2013. DigiTaps: Eyes-free Number Entry on Touchscreens with Minimal Audio Feedback. In Proceedings of the 26th Annual ACM Symposium on User Interface Software and Technology (UIST '13). ACM, New York, NY, USA, 85-90. DOI : http://dx.doi.org/10.1145/2501988.2502056

[7] Syed Masum Billah, Vikas Ashok, Donald E. Porter, and I.V. Ramakrishnan. 2017. Ubiquitous Accessibility for People with Visual Impairments: Are We There Yet? In Proceedings of the 2017 CHI Conference on Human Factors in Computing Systems (CHI'17). ACM, New York, NY, USA, 5862-5868. DOI :

http://dx.doi.org/10.1145/3025453.3025731

[8] Erin Brady, Meredith Ringel Morris, Yu Zhong, Samuel White, and Jeffrey P. Bigham. 2013. Visual Challenges in the Everyday Lives of Blind People. In Proceedings of the SIGCHI Conference on Human Factors in Computing Systems (CHI '13). ACM, New York, NY, USA, 2117-2126. DOI :

http://dx.doi .org/10.1145/2470654 . 2481291

[9] Virginia Braun and Victoria Clarke. 2006. Using thematic analysis in psychology. Qualitative research in psychology 3, 2 (2006), 77-101. DOI :

http://dx.doi.org/10.1191/1478088706qp063oa

[10] Maria Claudia Buzzi, Marina Buzzi, and Barbara Leporini. 2011. Web 2.0: Twitter and the Blind. In Proceedings of the 9th ACM SIGCHI Italian Chapter International Conference on Computer-Human
Interaction: Facing Complexity (CHItaly). ACM, New York, NY, USA, 151-156. DOI:

http://dx.doi.org/10.1145/2037296.2037333

[11] Yuri Choi, Kyung Hoon Hyun, and Ji-Hyun Lee. 2017. Image-Based Tactile Emojis: Improved Interpretation of Message Intention and Subtle Nuance for Visually Impaired Individuals. Human-Computer Interaction 0, 0 (2017), 1-30. DOI :

http://dx.doi.org/10.1080/07370024.2017.1324305

[12] Moodle Education Claudia Dent. 2017. Enhance your online communications through more support for emoji characters in Moodle 3.3.

https://moodle.com/news/enhance-online-

communications-support-emoji-characters-moodle-3-3. (2017). Accessed: 2019-09-04.

[13] Barry L Cole. 2004. The handicap of abnormal colour vision. Clinical and Experimental Optometry 87, 4-5 (2004), 258-275.

[14] Henriette Cramer, Paloma de Juan, and Joel Tetreault. 2016. Sender-intended Functions of Emojis in US Messaging. In Proceedings of the 18th International Conference on Human-Computer Interaction with Mobile Devices and Services (MobileHCI '16). ACM, New York, NY, USA, 504-509. DOI: http://dx.doi.org/10.1145/2935334.2935370

[15] Daantje Derks, Arjan ER Bos, and Jasper Von Grumbkow. 2007. Emoticons and social interaction on the Internet: the importance of social context. Comput hum behav 23, 1 (2007), 842-849.

[16] Jill P. Dimond, Casey Fiesler, Betsy DiSalvo, Jon Pelc, and Amy S. Bruckman. 2012. Qualitative Data Collection Technologies: A Comparison of Instant Messaging, Email, and Phone. In Proceedings of the 17th ACM International Conference on Supporting Group Work (GROUP '12). ACM, New York, NY, USA, 277-280. DOI :

http://dx.doi.org/10.1145/2389176.2389218

[17] Abdallah El Ali, Torben Wallbaum, Merlin Wasmann, Wilko Heuten, and Susanne CJ Boll. 2017. Face2Emoji: Using Facial Emotional Expressions to Filter Emojis. In Proceedings of the 2017 CHI Conference Extended Abstracts on Human Factors in Computing Systems (CHI EA '17). ACM, New York, NY, USA, 1577-1584. DOI : http://dx.doi .org/10.1145/3027063.3053086

[18] emojipedia. 2017. 5 Billion Emojis Sent Daily on Messenger. https://blog.emojipedia.org/5-billionemojis-sent-daily-on-messenger/. (2017). Accessed: 2019-08-28.

[19] Instagram Engineering. 2015. Emojineering Part 1: Machine Learning for Emoji Trends. https://instagram-engineering.com/emojineering-part-1machine-learning-for-emoji-trendsmachine-learningfor-emoji-trends-7f5f9cb979ad. (2015). Accessed: 2019-08-28. 
[20] World Health Organisation (WHO) Programme for the Prevention of Blindness and Deafness. 2003. Consultation on development of standards for characterization of vision loss and visual functioning. https://apps.who.int/iris/handle/10665/68601. (2003). Accessed: 2019-09-03.

[21] Justin Garrity. 2016. How Custom Emojis Crowned Twitter the Hashtag Registrar.

https://medium.com/@justinogarrity/how-customemojis-crowned-twitter-the-hashtag-registrar-

43e5fa60c557. (2016). Accessed:

2019-09-04.

[22] Cole Gleason, Patrick Carrington, Cameron Cassidy, Meredith Ringel Morris, Kris M. Kitani, and Jeffrey P. Bigham. 2019. "It's Almost Like They're Trying to Hide It": How User-Provided Image Descriptions Have Failed to Make Twitter Accessible. In The World Wide Web Conference (WWW'19). ACM, New York, NY, USA, 549-559. DOI :

http://dx.doi.org/10.1145/3308558.3313605

[23] Eric Goldman. 2018. Emojis and the Law. Wash. L. Rev. 93 (2018), 1227.

[24] Benjamin M. Gorman and David R. Flatla. 2017. A Framework for Speechreading Acquisition Tools. In Proceedings of the 2017 CHI Conference on Human Factors in Computing Systems (CHI'17). ACM, New York, NY, USA, 519-530. DOI : http://dx.doi.org/10.1145/3025453.3025560

[25] Tim Highfield and Tama Leaver. 2016. Instagrammatics and digital methods: Studying visual social media, from selfies and GIFs to memes and emoji. Communication Research and Practice 2, 1 (2016), 47-62.

[26] Albert H Huang, David C Yen, and Xiaoni Zhang. 2008. Exploring the potential effects of emoticons. Inform Manage 45, 7 (2008), 466-473.

[27] Unicode Inc. 2019a. Emoji Data for UTS. https://www.unicode.org/Public/emoji/12.0/emojidata.txt. (2019). Accessed:

2019-08-28.

[28] Unicode Inc. 2019b. Unicode ${ }^{\circledR}$ Full Emoji List, v12.0. (2019). Accessed: 2019-09-18.

[29] Unicode Inc. 2019c. Unicode ${ }^{\circledR}$ Technical Standard \#51 Design Guidelines. unicode.org/reports/tr51/\#Design_Guidelines. (2019). Accessed: 2019-09-18.

[30] (UTC) Unicode Inc. 2019d. Working Draft for Proposed Update Unicode ${ }^{\circledR}$ Technical Standard \#51. unicode.org/L2/L2019/19203r2-wd-uts51-17draft.pdf\#page=31. (2019). Accessed: 2019-09-18.

[31] Simon Keates John Clarkson, Roger Coleman and Cherie Lebbon. 2003. Inclusive Design: Designing for the Whole Population. Springer-Verlag London. DOI: http://dx.doi.org/10.1007/978-1-4471-0001-0
[32] Sigrid Kelsey and Kirk St. Amant. 2008. Handbook of Research on Computer Mediated Communication. IGI Global, Hershey, PA.

[33] Adam Kendon. 1967. Some functions of gaze-direction in social interaction. Acta Psychologica 26 (1967), 22 63. DOI: http://dx.doi.org/https: //doi.org/10.1016/0001-6918(67)90005-4

[34] Philippe Kimura-Thollander and Neha Kumar. 2019. Examining the "Global" Language of Emojis: Designing for Cultural Representation. In Proceedings of the 2019 CHI Conference on Human Factors in Computing Systems (CHI '19). ACM, New York, NY, USA, Article 495, 14 pages. DOI: http://dx.doi.org/10.1145/3290605.3300725

[35] Elizabeth Kirley and Marilyn McMahon. 2017. The emoji factor: Humanizing the emerging law of digital speech. Tenn. L. Rev. 85 (2017), 517.

[36] Matthew Knott. 2015. Emoji diplomacy? DFAT asked meaning behind Julie Bishop's use of a red-faced emoji to describe Vladimir Putin. Sydney Morning Herald, https://www.smh.com.au/politics/federal/emojidiplomacy-dfat-asked-meaning-behind-julie-bishopsuse-of-a-redfaced-emoji-to-describe-vladimir-putin20151022-gkfgoc.html. (2015). Accessed: 2019-09-04.

[37] Justin Kruger, Nicholas Epley, Jason Parker, and Zhi-Wen Ng. 2005. Egocentrism over e-mail: Can we communicate as well as we think? J. Pers. Soc. Psychol. 89, 6 (2005), 925-936. DOI :

http://dx.doi.org/10.1037/0022-3514.89.6.925

[38] Howard Lake. 2019. RNIB Aims to Redesign Emojis for Visually Impaired Users. fundraising.co.uk/2019/07/17/rnib-aims-to-redesignemojis-for-visually-impaired-users. (2019). Accessed: 2019-09-04.

[39] Weijian Li, Yuxiao Chen, Tianran Hu, and Jiebo Luo. 2018. Mining the Relationship between Emoji Usage Patterns and Personality. In Twelfth International AAAI Conference on Web and Social Media.

[40] Shao-Kang Lo. 2008. The nonverbal communication functions of emoticons in computer-mediated communication. CyberPsychology \& Behavior 11, 5 (2008), 595-597.

[41] Xuan Lu, Wei Ai, Xuanzhe Liu, Qian Li, Ning Wang, Gang Huang, and Qiaozhu Mei. 2016. Learning from the Ubiquitous Language: An Empirical Analysis of Emoji Usage of Smartphone Users. In Proceedings of the 2016 ACM International Joint Conference on Pervasive and Ubiquitous Computing (UbiComp '16). ACM, New York, NY, USA, 770-780. DOI : http://dx.doi.org/10.1145/2971648.2971724

[42] David F Marks and Lucy Yardley. 2004. Research methods for clinical and health psychology. Sage. 
[43] Nora McDonald, Sarita Schoenebeck, and Andrea Forte. 2019. Reliability and Inter-Rater Reliability in Qualitative Research: Norms and Guidelines for CSCW and HCI Practice. Proc. ACM Hum.-Comput. Interact. 3, CSCW, Article Article 72 (Nov. 2019), 23 pages. DOI: http://dx.doi.org/10.1145/3359174

[44] Hannah Miller, Daniel Kluver, Jacob Thebault-Spieker, Loren Terveen, and Brent Hecht. 2017. Understanding emoji ambiguity in context: The role of text in emoji-related miscommunication. In Eleventh International AAAI Conference on Web and Social Media.

[45] Hannah Jean Miller, Jacob Thebault-Spieker, Shuo Chang, Isaac Johnson, Loren Terveen, and Brent Hecht. 2016. "Blissfully Happy" or "Ready to Fight": Varying Interpretations of Emoji. In Tenth International AAAI Conference on Web and Social Media.

[46] Hannah Miller Hillberg, Zachary Levonian, Daniel Kluver, Loren Terveen, and Brent Hecht. 2018. What I See is What You Don'T Get: The Effects of (Not) Seeing Emoji Rendering Differences Across Platforms. Proc. ACM Hum.-Comput. Interact. 2, CSCW, Article 124 (Nov. 2018), 24 pages. DOI :

http://dx.doi.org/10.1145/3274393

[47] Sherwyn P Morreale, Brian H Spitzberg, and J Kevin Barge. 2006. Human communication: Motivation, knowledge, and skills. Cengage Learning.

[48] Meredith Ringel Morris, Jazette Johnson, Cynthia L. Bennett, and Edward Cutrell. 2018. Rich Representations of Visual Content for Screen Reader Users. In Proceedings of the 2018 CHI Conference on Human Factors in Computing Systems (CHI '18). ACM, New York, NY, USA, Article 59, 11 pages. DOI: http://dx.doi.org/10.1145/3173574.3173633

[49] Meredith Ringel Morris, Annuska Zolyomi, Catherine Yao, Sina Bahram, Jeffrey P. Bigham, and Shaun K. Kane. 2016. "With Most of It Being Pictures Now, I Rarely Use It": Understanding Twitter's Evolving Accessibility to Blind Users. In Proceedings of the 2016 CHI Conference on Human Factors in Computing Systems (CHI '16). ACM, New York, NY, USA, 5506-5516. DOI :

http://dx.doi.org/10.1145/2858036.2858116

[50] Leah Perlmutter, Bindita Chaudhuri, Justin Petelka, Philip Garrison, James Fogarty, Jacob O. Wobbrock, and Richard E. Ladner. 2019. Demonstration of GestureCalc: An Eyes-Free Calculator for Touch Screens. In The 21st International ACM SIGACCESS Conference on Computers and Accessibility (ASSETS '19). ACM, New York, NY, USA, 667-669. DOI :

http://dx.doi.org/10.1145/3308561.3354595

[51] Katy Perry. n.d. https://twitter.com/katyperry. (n.d.). Accessed: 2019-09-04.

[52] Henning Pohl, Christian Domin, and Michael Rohs. 2017. Beyond Just Text: Semantic Emoji Similarity
Modeling to Support Expressive Communication. ACM Trans. Comput.-Hum. Interact. 24, 1, Article 6 (March 2017), 42 pages. DOI :

http://dx.doi .org/10.1145/3039685

[53] Henning Pohl, Dennis Stanke, and Michael Rohs. 2016. EmojiZoom: Emoji Entry via Large Overview Maps. In Proceedings of the 18th International Conference on Human-Computer Interaction with Mobile Devices and Services (MobileHCI'16). ACM, New York, NY, USA, 510-517. DOI :

http://dx.doi.org/10.1145/2935334.2935382

[54] National Rail. n.d. https://twitter.com/nationalrailenq. (n.d.). Accessed: 2019-09-16.

[55] Maya Rhodan. 2014. President Obama Is Reaching Out to Millennials About the Economy Using...Emoji. https://time.com/3484876/obama-emoji/. (2014). Accessed: 2019-09-04.

[56] David Rodrigues, Diniz Lopes, Marília Prada, Dominic Thompson, and Margarida V. Garrido. 2017. A frown emoji can be worth a thousand words: Perceptions of emoji use in text messages exchanged between romantic partners. Telematics and Informatics 34, 8 (2017), 1532 1543. DOI: http://dx.doi.org/https:

//doi.org/10.1016/j.tele.2017.07.001

[57] Adrian Roselli. 2016. Accessible Emoji, Tweaked. http://adrianroselli.com/2016/12/accessible-emojitweaked.html. (2016). Accessed:

2019-03-18.

[58] Morgan Klaus Scheuerman. 2019. HCI Guidelines for Gender Equality and Inclusivity; updated 5/13/2019. https://www.morgan-klaus.com/sigchi-genderguidelines. (2019). Accessed: 2019-20-09.

[59] Andrew Sears and Vicki L. Hanson. 2012. Representing Users in Accessibility Research. ACM Trans. Access. Comput. 4, 2, Article Article 7 (March 2012), 6 pages. DOI: http://dx.doi .org/10.1145/2141943.2141945

[60] Katy Steinmetz. 2015. Oxford's 2015 Word of the Year Is This Emoji. TIME, https://time.com/4114886/oxfordword-of-the-year-2015-emoji/. (2015). Accessed: 2019-09-04.

[61] Gerhard Strube. 1987. Answering survey questions: The role of memory. In Social information processing and survey methodology. Springer, 86-101.

[62] Selina Sutton and Shaun Lawson. 2017. A Provocation for Rethinking and Democratising Emoji Design. In Proceedings of the 2017 ACM Conference Companion Publication on Designing Interactive Systems (DIS '17 Companion). ACM, New York, NY, USA, 7-12. DOI : http://dx.doi.org/10.1145/3064857.3079109

[63] Sarit Felicia Anais Szpiro, Shafeka Hashash, Yuhang Zhao, and Shiri Azenkot. 2016. How People with Low Vision Access Computing Devices: Understanding Challenges and Opportunities. In Proceedings of the 
18th International ACM SIGACCESS Conference on Computers and Accessibility (ASSETS '16). ACM, New York, NY, USA, 171-180. DOI :

http://dx.doi.org/10.1145/2982142.2982168

[64] Garreth W. Tigwell and David R. Flatla. 2016. Oh That's What You Meant!: Reducing Emoji Misunderstanding. In Proceedings of the 18th International Conference on Human-Computer Interaction with Mobile Devices and Services Adjunct (MobileHCI '16). ACM, New York, NY, USA, 859-866. DOI : http://dx.doi.org/10.1145/2957265.2961844

[65] Garreth W. Tigwell, David R. Flatla, and Rachel Menzies. 2018. It's Not Just the Light: Understanding the Factors Causing Situational Visual Impairments During Mobile Interaction. In Proceedings of the 10th Nordic Conference on Human-Computer Interaction (NordiCHI '18). ACM, New York, NY, USA, 338-351. DOI : http://dx.doi.org/10.1145/3240167.3240207

[66] Garreth W. Tigwell, Zhanna Sarsenbayeva, Benjamin M. Gorman, David R. Flatla, Jorge Goncalves, Yeliz Yesilada, and Jacob O. Wobbrock. 2019. Addressing the Challenges of Situationally-Induced Impairments and Disabilities in Mobile Interaction. In Extended Abstracts of the 2019 CHI Conference on Human Factors in Computing Systems (CHI EA '19). ACM, New York, NY, USA, Article W30, 8 pages. DOI:

http://dx.doi.org/10.1145/3290607.3299029

[67] Sarah J. Tracy. 2013. Qualitative Research Methods: Collecting Evidence, Crafting Analysis, Communicating Impact. Wiley-Blackwell.

[68] Unicode. 2019. Members - Unicode. https://home.unicode.org/membership/members/. (2019). Accessed: 2019-09-18.

[69] Veroniiiica. 2018. How Do People with Vision Impairments...Use Emoji?

http://www.perkinselearning.org/technology/blog/howdo-people-vision-impairments-use-emoji. (2018). Accessed: 2019-03-18.

[70] Joseph B Walther and Kyle P D' Addario. 2001. The impacts of emoticons on message interpretation in computer-mediated communication. Social science computer review 19, 3 (2001), 324-347.
[71] Léonie Watson. 2016. Accessible emoji. https://tink.uk/accessible-emoji/. (2016). Accessed: 2019-03-18.

[72] Brian Wentz, Harry Hochheiser, and Jonathan Lazar. 2013. A survey of blind users on the usability of email applications. Universal Access in the Information Society 12, 3 (01 Aug 2013), 327-336. DOI:

http://dx.doi .org/10.1007/s10209-012-0285-9

[73] World Health Organisation (WHO). 2018. Blindness and vision impairment. https://www.who.int/news-room/factsheets/detail/blindness-and-visual-impairment. (2018). Accessed: 2019-09-04.

[74] Sarah Wiseman and Sandy J. J. Gould. 2018. Repurposing Emoji for Personalised Communication: Why Pizza Slice Emoji; Means "I Love You". In Proceedings of the 2018 CHI Conference on Human Factors in Computing Systems (CHI'18). ACM, New York, NY, USA, Article 152, 10 pages. DOI : http://dx.doi.org/10.1145/3173574.3173726

[75] Shaomei Wu and Lada A. Adamic. 2014. Visually Impaired Users on an Online Social Network. In Proceedings of the SIGCHI Conference on Human Factors in Computing Systems (CHI '14). ACM, New York, NY, USA, 3133-3142. DOI : http://dx.doi.org/10.1145/2556288.2557415

[76] Peijun Zhao, Jia Jia, Yongsheng An, Jie Liang, Lexing Xie, and Jiebo Luo. 2018. Analyzing and Predicting Emoji Usages in Social Media. In Companion Proceedings of the The Web Conference 2018 (WWW '18). International World Wide Web Conferences Steering Committee, Republic and Canton of Geneva, Switzerland, 327-334. DOI :

http://dx.doi.org/10.1145/3184558.3186344

[77] Rui Zhou, Jasmine Hentschel, and Neha Kumar. 2017. Goodbye Text, Hello Emoji: Mobile Communication on WeChat in China. In Proceedings of the 2017 CHI Conference on Human Factors in Computing Systems (CHI '17). ACM, New York, NY, USA, 748-759. DOI : http://dx.doi.org/10.1145/3025453.3025800 